\title{
Die Preise im Konjunkturverlauf
}

\author{
Von J. Tinbergen, Scheveningen
}

Vom Österreichischen Institut für Konjunkturforschung ist, gemeinsam mit der London School of Economics, eine Arbeit von Gerhard Tintner herausgegeben worden ${ }^{1}$ ), in der eine sehr große Anzahl statistischer Daten bezüglich des Verhaltens der Preise im Konjunkturzyklus zusammengebracht worden ist. Dex Verfasser hat etwa hundert deutsche Preise und ebensoviele englische und amerikanische, nebst einigen österreichischen, holländischen usw. gesammelt, von denen eine Anzahl bis 1845 zurückgehen und die im allgemeinen bis 1913 reichen; alles Monatsreihen. Weitaus die Mehrzahl derselben sind Güterpreise; außerdem noch einige Fracht- und Zinsraten. Löhne wurden nicht bearbeitet.

Zunächst werden die zufälligen Komponenten dieser Preise nach Andersons (Gossets) "variate difference method" eliminiert. Es ergibt aich, dab im Allgemeinen mit ungewogenen gleitenden Mittelwerten gearbeitet werden kann, deren Länge zwischen 11 und 3 Monaten zu wechseln hat, falls die Reste der zufälligen Komponenten nicht mehr als im Mittel 2\% der Werte betragen sollen.

Sodann werden die Saisonschwankungen durch gleitende 12-Monatsmittelwerte ansgeschaltet, und von den so bearbeiteten Reihen durch Anwendung gleitender Mittelwerte mit veränderlicher Länge, je nach der Dauer der Zyklen, die Trends bestimmt. Durch Subtraktion dieser Trendkomponente erhält man die zyklische Komponente, die das eigentliche Untersuchungsobjekt bildet.

Tintner gibt eine sehr sorgfältige Begründung der bekannten, seiner Analyse zugrunde liegenden Auffassung, es könne die von Zufallseinflüssen bereinigte statistische Reihe in drei (additive) Komponenten: Saison-, Trendund zyklische Komponente zerlegt werden. Er ordnet die Ursachenreihen in drei Gruppen mit je einer Gesamtreihe ein, bei der die zu der Gruppe gehörigen Zahlen eben in der Weise kombiniert worden sind, wie sie auf die betrachtete Reihe wirken. Wenn diese Gesamtreihen mit $s, t$ und $k$ angedeutet werden, so nimmt Tintner zuerst allgemein an, daß die mathematische Erwartung der betrachteten Reihe $x$ eine ganz allgemeine Funktion von $s, t$ und $k$ sei. Diese Funktion zerlegt er dann in eine Taylor-Maclanrinsche Reihe, die er nach den linearen Gliedern abbricht. So erhält er als fast allgemeingültige Annäherung die lineare Kombination

$$
x=f_{0}+f_{s} s+f_{t} t+f_{k} k
$$

wobei die $f_{0}, f_{s}, f_{t}$ und $f_{k}$ Konstanten sind. Er schreibt dann für

$$
f_{s} s=\mathbb{S}, f_{0}+f+t=T, f_{k}=K,
$$

3) Dr. Gerhard Tintner, Prices in the Trade Cycle, published in cooperation with the London School of Economies and Political Science by the Austrian Institute for Trade Cycle Rcsearch. With a Foreword hy Oskar Morgenstern; Vienna 1935 (Jullus Springer). 
wodurch die bekannte Zerlegung erreicht wird:

$$
x=T+K+S \text {. }
$$

Ich habe hiezu nur eine kleine terminologische Bemerkung zu machen; es entspricht meines Erachtens der Zweckmäßigkeit und Logik, daß man den Namen „Komponenten" nur für $T, K$ und $S$, und nicht auch für $8, t$ und $k$ benutzt.

Die eigentliche Untersuchung betrifft in mannigfacher Weise das zyklische Verhalten der Preise. Dieses wird in sehr vielen statistischen Mittelwerten dargelegt. Tintner berechnet die Prozentzahl der Monate in welcher eine Preisänderung auftritt, den Prozentsatz aller Konjunkturphasen, an welchen die betreffende Reihe teilnimmt, die mittlere Länge des Zyklus, den Anteil der Periode der steigenden Preise am ganzen Zyklus, die Amplitude (in Prozenten des Trends), den Unterschied der Amplituden der Konjunkturbewegungen zwischen Hoch- und Tiefpunkt und die mittlere Aufeinanderfolge der Preise im Hoch- und Tiefpunkt. Diese Zahlen werden sowohl für die einzelnen Preise, wie auch für gewisse Preisgruppen berechnet. Die Gruppen wieder werden nach verschiedenen Gesichtspunkten aufgebaut; z. B. werden folgende Gruppen gebildet: Brotgetreide, Ól und Fett, Vieh und Fleisch, Nahrungsmittel, Genußmittel, europäische Metalle, amerikanische Metalle; Betriebsstoffe (europäische und amerikanische), Textilrohstoffe und -produkte, Maschinen, Werkzeuge usw. Es werden noch umfassendere Gruppen gebildet, z. B. Preise tierischer, solche pflanzlicher und solche anorganischer Güter, arbeitsintensiver und kapitalintensiver Güter, Preise von Rohstoffen, Halbfabrikaten und Konsumgütern, von spezifischen, halbspezifischen und unspezifischen Gütern.

Von den Resultaten seien nur die allerwichtigsten erwähnt: für die vielen wertvollen Details möge der Leser die Publikation selbst nachschlagen! Ganz allgemein stellt der Verfasser fest, daß , die Konjunkturbewegungen... nneinheitlicher und mannigfaltiger (sind), als man gemeinhin denkt". Die mittlere Länge der Zyklen ist relativ kurz bei Textilgütern, beim Zins, bei den Frachten, den tierischen Rohstoffen, den arbeitsintensiven, den Ernteund den konsumreifen Gütern und den unspezifischen Produktionsmitteln. Relativ lange Zyklen finden sich bei den Metallen, den kapitalintensiven und den kontinuierlich produzierten Gütern und den Halbfabrikaten.

Eine große mittlere monatliche Veränderlichkeit zeigen u. a. der Zinsfub, die Textilindustrie und die Erntegüter.

Wir sehen ein Vorausgehen bei den landwirtschaftlichen Preisen, ein Nacheilen der kontinuierlich produzierten und der kapitalintensiven Güter.

Ein internationaler Vergleich zeigt, daß England und Deutschland einen viel engeren Zusammenhang als Amerika und Europa zeigen.

Der Verfasser schreibt weiter, daß ,gewisse Momente... die Vermutung nahe (legen), daß der Zinsfuß und die Preise der mehr oder weniger spezifischen Produkte und der Produktionsmittel eine große Rolle im Konjunkturzyklus spielen" (S.93, wo auch die übrigen Resultate zusammengefaßt werden). Der Verfasser beendet die Aufzählung der Resultate mit der Frage, „ob nicht eine Konjunkturtheorie, welche den Konjunkturzyklus nicht auf eine einzige Ursache, sondern auf mehrere versehiedene Ursachen zurückführt, uns dieses uneinheitliche Verhalten erklären könnte". Darin kann ich ihm nur voll und ganz beistimmen.

Nach dieser kurzen Darstellung des Inhalts des Buches seien einige kritische Bemerkungen über die Bedeutung der gebrauchten Methode und der angegebenen Resultate hinzugefügt. 
Was erstens die Methode anbetrifft, sei bemerkt, daB ich mit Tintners Auffassung der Rolle der Statistik und der ökonomischen Wissenschaft in der Forschung vollkommen einverstanden bin. Tintner sieht die Statistik ganz zu Recht als Hilfe und Kontrolle der ökonomischen Theorie an. Ich möchte bemerken, daß er, meinem Geschmack nach, doch noch immer etwas zu sehr statistisch und etwas zu wenig ökonomisch gearbeitet hat. Ich meine damit nicht, daß ich der gewählten Zerlegungsmethode nicht beistimme im Gegenteil -; sondern die in der ,eigentlichen Untersuchung“, wie ich sie oben genannt habe, befolgte Arbeitsweise zeigt Spuren eines zu starken Vorwiegens der statistischen Analyse.

Das einfachste Beispiel, das ich geben kann, ist wohl das folgende. Soweit ich sehe, sind die Schwankungen der Erntegüterpreise in starkem Maße durch die Ernteschwankungen bestimmt. Die bei ihnen auftretenden Zyklen sind meines Erachtens nur wenig - oder überhaupt nicht - mit den Schwankungen von echten konjunkturempfindlichen Waren, wie vor allem von Metallen und deren Produkten zu vergleichen. Nur bei den Textilrohstoffen etwa liegt ein Mischverhältnis vor. Es ist meines Erachtens dann auch zweifelhaft, ob das Voreilen der Preise der Erntewaren viel Bedeutung hat. Hier scheint mir eine Korrelationsanalyse wertvoll, die den Zusammenhang dieser Preise mit den Ernte- und Vorratsmengen einerseits und mit Konjunkturanzeigern wie den Investitionsvolumen oder den Einkommen anderseits untersuchen würde. $\mathrm{Ob}$ ein solches Voraneilen der agrarischen Preise Bedeutung hat, könnte man auch nicht nur nach einem Mittelwert dieses Lags beurteilen, - was übrigens auch Tintner hervorhebt - sondern nur nach der Natur der betreffenden Reihen selbst. Nebenbei sei bemerkt, dab vom graphischen Hilfsmittel mehr Gebrauch hätte gemacht werden können. Hiebei hat der Verfasser vielleicht etwas zu wenig mit der Faulheit der Leser gerechnet.

Das merkwürdige Resultat, daß die Metallpreise „nicht an allen Zyklen teilnehmen"s, wird durch obige Bemerkungen anch in etwas anderer Weise verständlich. Es liegt die Frage nahe, ob nicht gerade die Metallpreise die ",reellen" Zyklen zeigen, während die kleineren Zyklen bei den Erntewaren anderer Natur sind, als der, die wir in der Konjunkturforachung den Zyklen zuschreiben ${ }^{1}$ ).

Nach dieser Vorbemerkung komme ich auf die Methoden selbst. Wie schon gesagt, wird die Voranalyse äußerst sorgfältig vorgenommen, wofür man dem Autor deswegen dankbar sein muB. Sein Material ist auch für andere Zwecke verwendbar. Bei der weiteren, der ,eigentlichen " Untersuchung, wird auch manche wichtige Berechnung unternommen. So z. B. die Untersuchung des unterschiedlichen Verhaltens der Preise in der Hochkonjunktur und in der Depression. Andererseits habe ich anch hier wieder einige Bedenken. Erstens ist es mir kaum verständlich, wie man die Zahl der Zyklen feststellen kann. Mancher Zyklus zeigt ganz kleine Unterbrechungen. Diese wird man offenbar nicht als einzelne Zyklen mitzählen, obgleich sie, genau genommen, ein relatives Maximum und ein relatives Minimum verursachen. Wie aber bei den größeren Unterbrechungen? Die Grenze zwischen mitzuzählenden and nicht mitzuzählenden Zyklen ist durchaus unscharf. Denkt man sich den Konjunkturverlauf wie Frisch; als die Bewegung eines durch zufällige

1) Ich könnte meinen Einwand auch so formulieren, daf die Jahresschwankungen der Preise der Ernteguter eigentlich auch zufälliger Natur sind und also auch ausgeschaltet werden sollten. Das öfnet weitere Problemkreise einer vielleicht etwas $z \mathbf{u}$,mechanischen Glattung:" 
Störungen von außen her bewegten Systems, das an und für sich streng gesetzmäßige Bewegungen aufweist, so erhält man durch das Zählen der relativen Maxima und Minima gar keinen richtigen Eindruck der Wellenlänge, falls mittelgroße und große Störungen auftreten. Zur Illustration sei die Abbildung betrachtet. Die Bewegung eines Systems sei streng periodisch, falls keine neuen, äußeren Störungen darauf einwirken. Die Wellenlänge sei $T$. Falls nun in $A$ und $B$ diese ,natürliche" Bewegung unterbrochen wird, so kommen relative Maxima und Minima hinzu, die für die Messung der Periode keine Bedeutung haben.

Ein zweiter Einwand betrifft den Begriff der mittleren monatlichen Veränderung, die u. a. beim Zins besonders groß ist. Sie wird nämlich in Prozenten des Trendwertes ausgedrückt, und daher ist es einleuchtend, daß man einen ganz anderen Wert erhalten würde, falls man statt des Preises der Kapitalsdisposition den Preis gegenwärtiger Güter (z. B. in Gütern des nächsten Jahres ausgedrückt) betrachtet hätte. Ist der Zinsfuß $5 \%$, so ist der letztere Preis 105 und die Schwankungen in Prozenten dieses letzten Wertes sind natürlich viel geringer.

Eine weitere Bemerkung betrifft die Art, wie der Verfasser die internationalen Vergleiche durchführt. Auch hier hätte er meines Erachtens mit Erfolg von der Korrelationsrechnung Gebrauch machen können. Der Grad der internationalen Verbundenheit kann sehr leicht durch den Korrelationskoeffizienten $r$ oder durch $\sqrt{1-r^{2}}$

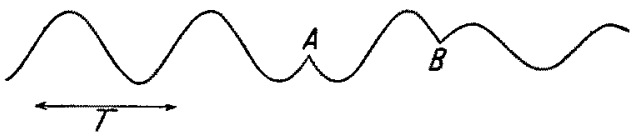

Abb. 1. wiedergegeben werden. Die vom Verfasser gewähiten Methoden scheinen dagegen ziemlich verwickelt zu sein.

Wenden wir uns jetzt den von Tintner gezogenen Schlüssen zu. Im Allgemeinen drückt er sich sehr vorsichtig aus; er ist sich der Vorwürfe wahrscheinlichkeitstheoretischer Natur durchaus bewußt, die bei einer allzugewissen Ausdrucksweise hie und da erhoben werden könnten. Verschiedene der Schlußfolgerungen werden den Leser nicht verwundern: sie bestätigen Meinungen, die man auch auf andere Weise erreicht hat. Bei den Schlußfolgerungen bezüglich der internationalen Verbundenheit der Preise hätte man noch bemerken können, - wenn ich richtig sehe, - dah die Gewichtsmenge pro Werteinheit einen deutlichen Einfluß hat: je schwerer ein Gut im Verhältnis zu seinem Werte ist, um so schwieriger wird sein Transport, und um so schlechter wird der Ausgleich der Preise sein. Selbstverständlich spielen auch andere Momente eine Rolle.

Bei den interessanten Feststellungen über die Form der Schwankungen hätte vielleicht noch bemerkt werden können, daß diejenigen Preise, die die schärfsten Maxima zeigen, wahrscheinlich am stärksten die Amplitude der Konjunkturschwankungen der ganzen Wirtschaft bestimmen. Falls nämlich die Eisenpreise scharfe Maxima und z. B. die Weizenpreise runde Maxima zeigen, so ist es wahrscheinlich, daß eine Vergrößerung der Amplitude der Zyklen durch die Unmöglichkeit, mehr Eisen pro Monat zu produzieren, mehr erschwert wird, als durch die Unmöglichkeit, mehr Weizen pro Monat zu liefern. Entsprechend diesem Gedankengang könnte man also einigermaßen feststellen, wo die amplitudenbestimmenden Faktoren der Wirtschaft liegen und welche der diesbezüglichen Theorien bestätigt werden können. Die Theorie, daß Mangel an organischen Rohstoffen Ursache des Umsehlags bilde, scheint mir z. B. im Widerspruch zu den relativ runden Maxima der 
Preise dieser Artikel zu stehen. Ebenso die Theorie des Arbeitermangels: auch die Löhne zeigen keine scharfen Spitzen. Dagegen würde eine Theorie des Mangels an mineralischen Rohstoffen schon mehr mit der Wirklichkeit übereinstimmen. Ich muß gestehen, daB es mich überrascht hat, daB der Zinsfuß nicht schärfer zugespitzte Tiefpunkte aufweist; an den unkorrigierten Bildern hatte ich immer das Gegenteil zu sehen geglaubt. Ich hatte dieses Gegenteil sogar als eine gewisse Stütze für die Theorie des Kapitalmangels angesehen. Ich bin nicht ganz sicher, daß nicht diese Schlußfolgerung Tintners (S. 66, nämlich, daß die Zinsrate eine größere Amplitude in der Depression, als in der Hochkonjunktur zeigt) eine Folge der Ausschaltungsweise ,zufälliger" Störungen ist. Es könnte sein, daß durch die gleitenden Mittelwerte Spitzen abgeschnitten werden, die ausnahmsweise nicht zufälliger Natur sind, nämlich die Zinsenspitzen in der Krise. Es wäre wichtig, hierüber Tintners Meinung zu hören.

Hiemit sind wir auch auf den Umstand zu sprechen gekommen, der mich, und mit mir wahrscheinlich mehrere Leser, am stärksten beeindruckt hat, nämlich die an verschiedenen Stellen erwähnte Folgerung, daß der Zingfuß im Konjunkturverlauf eine große Rolle spiele, - ein Umastand, der die monetären Theorien mehr oder weniger unterstützt. Es sei bemerkt, daß sich Tintner auch hier sehr vorsichtig ausdrückt. Ich verstehe aber die Argumente, die er anführt, nicht.

Es ist auch schon nicht genau zu begreifen, was ,eine Rolle spielen“" hier bedeutet. Meines Errachtens soll es heißen, daß im Mechanismus der Wellen, - im Kausalnexus der Wirkungen, die die Wellen hervorrufen -, der Zinsfuß eine große Stelle einnimmt; daß er also etwa auf die Produktionsmenge, oder, besser noch, auf die Investitionsmenge einen sehr deutlichen Einfluß hat.

Worans folgert der Autor nun eine solche Sachlage? S. 58 wird erwähnt, daß der Zinsfuß die stärkste mittlere monatliche Veränderung zeigt. Auf S. 67 weiter, daß der Zinsfuß wesentlich früher in den Aufschwung als in den Abschwung geht, ..., , a fact which gives further support to the monetary theory". Falls ich nicht wichtige andere Stellen übersehen habe, sind diese zwei Stellen die Hauptgründe der angeführten These. Es wäre sehr wichtig, hierüber Näheres zu hören. Ich möehte, um die Diskussion auf die Spitze zu treiben, absichtlich die genau entgegengesetzte These entwickeln, nämlich daß der Verlauf des Zinsfußes für die Konjunkturbewegung in dem von mir soeben umschriebenen Sinne nicht wichtig sei. Ich darf das vielleicht durch Gebrauch eines Begriffes verdeutlichen, der mir gute Dienste beim Verstehen gewisser Konjunkturprobleme geleistet hat ${ }^{1}$ ), nämlich durch den Begriff der passiven oder symptomatischen Variablen. Unter einer solchen Variablen verstehe ich eine, die zwar vom Konjunkturmechanismus mitgezogen wird (und also die Konjunkturbewegungen mitmacht), aber selbst keinen Einfluß auf den Mechanismus hat, also auch nicht als Ursache weiterer Kausalkelten auftreten kann. Bei solchen Größen macht es daher nichts aus, ob sie die Konjunkturbewegung nun schwach oder stark zeigen; sie spielen bei ihrer Vorarsachung einfach keine Rolle. Der Hinweis Tintners auf die starke Beweging des Zinsfußes besage also nichts. Dies könnte meines Erachtens nur auf Grund multipler Korrelationsverfahren bewiesen werden, die zeigten, daß die Bewegungen des Investitionsvolumens in starkem Maß durch die Bewegungen des Zinsfußes bestimmt werden könnten. Erst dadurch könnte die „Passivitätt" oder ,Aktivität" (in meiner Terminologie) geprüft werden.

1) Vgl. Econometrica III (1935), S. 292. 
Meine bisherige Erfahrung auf dem Gebiet solcher Korrelationsrechnungen hat mich zur Einsicht geführt, daß die Zinsrate ziemlich unwichtig ist.

Ubrigens bin ich auch gar nicht sicher, daß die Theorie Wicksells wirklich behauptet, daß der Zinsfuß eine große Rolle spielt. Der Abstand zwischen natürlichem und Geldzins spielt nach dieser Theorie eine große Rolle. Wahrscheinlich sind aber die Bewegungen des natürlishen Zinses für die Bewegungen des Abstandes weitaus wichtiger, als die Bewegungen des Geldzinses. (Tintner betrachtet übrigens auch nur die ,kurze" Zinsrate, die gar nicht zur langen parallel verläuft.) Man bedenke bei alledem, daß in einer Wirtschaft mit einem unendlich elastischen Kreditsystem (wo also die von der monetären Theorie betonten Momente erst reeht stark auftreten würden) die Zinsrate sich ulberhaupt nicht bewegen würde und also als Ursache der Bewegungen ganz ausscheiden müßte!

Was das zweite von Tintner angeführte Argument betrifft, so muB ich gestehen, daß ich dessen Tragweite nicht übersehe. Man möchte gern bewiesen sehen, daß die vom Verfasser gefundene Sachlage mit nicht-monetären oder gemischten Theorien im Widerspruch stehe, bevor man einsehen kann, daß dieselbe ,eine weitere Stütze für die monetäre Theorie bildet". Auch hier wäre es gut, des Verfassers Meinung ausführlicher dargelegt zu lesen.

Hiemit habe ich alle kritischen Bemerkungen gemacht, zu denen die Lektüre des Buches mir Anlaß gibt, heißt es doch: „Indes ihr Komplimente drechselt, kann etwas Nützliches geschehn“. Diese kritischen Bemerkungen dürfen aber keineswegs milverstanden werden. Und deshalb möchte ich es noch kurz aussprechen, daß wir Tintner für die Fülle gewissenhafter und sorgfältiger Arbeit und das umfangreiche Material, das er uns gebracht hat, recht dankbar sind, zumal er es auch in sehr übersichtlicher Weise wiedergegeben hat. 